\title{
CommonSpaces: an approach to web learning based on OERs, mentoring and collaborative learning
}

\section{Lariccia, Stefano $^{a}$; Ritella, Giuseppe ${ }^{\mathrm{b}}$; Montanari, Marco ${ }^{\mathrm{a}}$; Cesareni, Donatella ${ }^{\mathrm{c}}$ and Toffoli, Giovannid}

${ }^{a}$ Department of European, American and Intercultural Studies, University of Rome Sapienza, Italy, ${ }^{\mathrm{b}}$ Institute of behavioral sciences, University of Helsinki, Finland ${ }^{c}$ Department of social and developmental psychology, University of Rome Sapienza, Italy; ${ }^{\mathrm{c}}$ Link SRL, Rome, Italy.

\begin{abstract}
Currently, the internet is full of freely accessible resources that can provide excellent learning opportunities. However, these resources are usually not well organized, and for many users it is often difficult to use these dispersed sources of knowledge in a coordinated way. The project CommonS aims at building a space - called CommonSpaces - dedicated to communities of practice in which participants learn through the cataloguing, re-use, adaptation and sequencing of Open Educational Resources (OERs) into socalled learning paths. We define a Learning Path as an organized set of interconnected OERs (created by communities of users) that can be created by users both to organize their learning experience and to provide consistent learning sequences for others. In this paper, we briefly present the rationale and the theoretical foundations of the project. Then we discuss the features of CommonSpaces in its first prototype version and describe the preliminary findings from a pioneering experience of collaborative learning carried out by means of CommonSpaces. We conclude discussing the ongoing collective modeling of the final version of CommonSpaces and its future directions.
\end{abstract}

Keywords: Web Learning, Open Educational Resources; Mentoring; Collaborative Learning; Learning Design; Technology-mediated Learning. 


\section{Introduction}

The process of digitalization is carrying profound changes in our lives, including the way in which we learn, the way we teach, the way we build new knowledge. Even the common sense understanding of space and time is changing under the pressure of digitalization (Ritella et al., submitted). The availability of multiple types of web spaces (semantic, pragmatic, cloud spaces) transforms the context of learning, and in turn, requires new ways of organizing learning practices. Learning through "digital contents" is a different activity than using a book. As an example, doing a literature review using google books or google scholar or other digital tools is radically different than visiting a traditional library (Ritella \& Hakkarainen, 2012). Our relationship with knowledge changes, and our approaches to learning should change accordingly. As suggested by Hakkarainen (2009) technology enhances learning only through transformed learning practices. We refer here also to the new mix of rules, techniques, and foundational principles that characterize the "web learning" framework (referring to the Web Sciences of T.Berners-Lee, 2006).

New attitudes are already at work in the adoption of new methodologies for knowledge building in schools and universities; but in many cases there is a lack of systematicity, a lack of awareness concerning this deep innovation process. In order to avoid this risk, a multidisciplinary effort is required, able to address the multiple aspects involved. For this reason, in our project we teamed up computer specialists with pedagogists, psychologists, and Natural Language Processing experts.

With the project CommonS we want to move some steps forward toward such digital evolution in learning and education. In our project, we are designing digital tools that will provide specific support for digital learning practices, based on the pedagogical model described in the next section. In particular, we wish to capitalize on the potentially infinite resources freely available on the internet, often labeled as Open Educational Resources (OERs). We created a web space for gathering some new tools for learning through OERs: some of these will be internally bundled (embedded tools), others will be only selected and suggested to our communities.

In the next session we will describe the theoretical framework and pedagogical model on which the project is based. Then we discuss the features of CommonSpaces in its first prototype version and describe the preliminary findings from a pioneering experience of collaborative learning carried out by means of CommonSpaces. We conclude discussing the ongoing collective modeling of the final version of CommonSpaces and its future directions. 


\section{The background of CommonSpaces}

CommonSpaces will represent the final output of the project CommonS. It is a space dedicated to communities of practice (Wenger et al., 2002) in which participants learn through the cataloguing, re-use, adaptation and sequencing of OERs into so-called learning path. Currently, the internet is full of freely accessible resources that can provide learning opportunities based on excellent learning contents, tools, platforms or a mix of these ingredients. However, these resources are usually not well organized, and for many users it is often difficult to use these dispersed sources of knowledge in a coordinated way. The risk is that the learner engages into shallow internet surfing, limiting the learning experience to the reading of separated chunks of knowledge. Therefore, one fundamental aspect of the project is to provide a consistent way to catalogue, organize and reuse them in coherent sequences on a given topic, that is, learning paths. We define a Learning Path as an organized set of interconnected OERs that can be created by users both to organize their learning experience and to provide consistent learning sequences for others.

The pedagogical model of CommonSpaces is based on some foundational principles that were abstracted from existing literature. First, some approaches to learning - such as Knowledge Building (Scardamalia \& Bereiter, 2006) and Trialogical Learning (Hakkarainen \& Paavola, 2009) - consider knowledge as a collective product that can be constructed and manipulated by the learner in multiple ways. The learner is called to actively work with sources of knowledge rather than passively accepting them as given; to develop soft skills such as communicative competence and critical thinking, in order to collaborate with their peers in building knowledge together. In this process, the teacher is expected to act as a coach other than a lecturer. Indeed, in these approaches, the role of the expert goes beyond the presentation of contents to the learner.

In CommonSpaces, the role of teacher, or experts in general, is to provide a scaffolding, guiding the students in the creation of their learning path. Therefore, another important aspect of the project is the development of a system of online mentoring through which experienced participants - who are granted the role of mentors - can support other participants in building their learning paths.

Finally, CommonSpaces intends to go beyond the individual use of OERs. By means of the provision of social interaction tools embedded in the platform and throught the work of the community manager and the project leaders, the projects aims at enhancing the networking between young and experienced professionals, and at the creation of communities of practice for professional development. 


\section{CommonSpaces: a web-space for collaborative learning based on OERs}

In our project, we designed and developed a technological infrastructure - CommonSpaces aimed at enhancing the learning processes based on OERs and implementing the pedagogical model described above. This space is organized around communities. Each community is composed by a variable number of members and a community manager. The latter can give members the possibility to form sub-groups focused on single projects with at least an internal supervisor who can be different from the community manager. Within each "project", the supervisors are able to accept membership, create new sub-projects, embed tools like forum or chat, send e-mails to all the project's participants and so on.

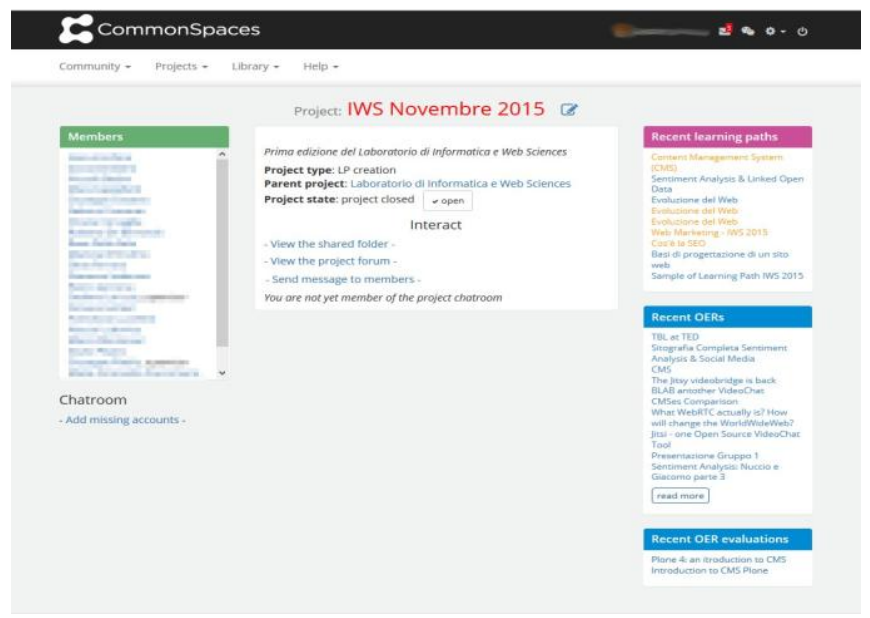

Figure 1. Screenshot from a project within CommonS

Fundamentally, projects (see Figure 1) represent the basic bricks of the whole space. CommonSpaces currently allows the creation of three types of projects:

1) OER cataloguing project: this project allows a group of learners interested in the same topic to gather OERs, add metadata and evaluate them collectively. The result is an organized database of OERs that can be easily accessible and manipulable;

2) Learning path creation project: this project allows a group to further organize the OERs in structured sequences that can be easily visualized and manipulated directly within the space, in order to reach pedagogical goals that go beyond the use of single OERs. For example, a learning path might contain fragments of video lessons, various kinds of documents and multimedia concerning a given topic. In this type of projects, participants can define relations between OERs, organizing them for a coherent learning experience; 
3) Mentoring projects: this project involves only two participants (a mentor and a mentee). It is meant to support the building of a one to one relationship between an expert and a novice, so that the experienced participant (i.e., the mentor) can guide and support the learning experience of the novice (i.e. the mentee) within CommonSpace.

For all of these types of projects, Commonspaces also provide communication tools and the assistance of the project leader and community manager for enhancing social interaction within the community. In order to design CommonSpaces in line with the principles presented in this paper, the technical choices to be addressed were not easy. The selection of the environment was made during a period of almost 2 years before the real start of the project. We initially were looking at Plone, an established Content Management System (CMS) written in Python. After a long period of evaluation we finally preferred to keep the choice of Python but we opted for a different web application framework, Django, integrated with a number of optional libraries to cope with some specific functionality.

We operated this choice to maintain the basic framework as simple as possible in order to be able to steer the development of the "space" forward the direction that the stakeholders of the project ("the communities") would have been defining with their "framework modeling". Rather than proposing a full set of ready to use functionalities, we preferred to let the partners express their needs step by step. This "style" of design might be defined as "collective incremental developing". We are putting in this definition the result of many experiences of the past decades, when we developed too complex framework following passively stakeholders functional needs. The partnership is guaranteed about the sustainability of the software package since the code is uploaded and updated on GitHub, a code sharing and publishing service.

The project foresee three stages of development and 3 Prototypes: a first prototype focused on OER collective classification; a second prototype handling mentoring relations and the ability to create Learning Path re-using the bricks of OER; a third prototype dealing with the integration of tracking / monitoring capabilities of the members activities inside the "CommonSpaces". Currently we are working on the second prototype. The final version will be integrated to bring new functionalities to community and members: functionalities aiming at shaping CommonSpaces more and more as a "learning design environment", an "authoring" and "auditing" tool for learners and teachers / tutors / mentors. Some of the functionalities that we are discussing are listed as follows: exporting LP into other formats (Moodle, Scorm, e-book); definition and implementation of a set of trackable data to monitorize collaborative activities; the light integration of a sophisticated form of video interaction supporting a living archive of registered sessions. The collective incremental style is foreseen to guide also the stage after the 3th prototype: after prototype 3 . we foreseen to open an "open coding contribution" where codlet and new libraries could be 
added by communities and integrated (by the software keepers) into the main structure of the CommonSpaces.

\section{CommonSpaces applied: a pioneering experience of collaborative learning}

At present there are 7 main communities within CommonSpaces. The community "Sapienza. University of Rome 1" is working on gathering more members through the activation of new projects that students can find useful for their professional development. The first project realized was called "Workshop on Informatics and Web Sciences". It was realized in November and December 2015 for a total of five meetings of three or four hours each. The workshop involved a small group of 24 students attending a Psychology master degree (in Italian: Laurea Magistrale) who volunteered to participate. The aims of the workshops were: 1) teaching some skills in computer science through the cataloguing, reuse, adaptation and sequencing of OERs; 2) reinforcing students' collaborative skills; 3) testing CommonSpaces on a technical base, 4) testing a set of didactic guidelines to use it.

The pedagogical approach adopted was derived from the theoretical framework described above. In sum, the students were invited to collaborate with each other in building a collective learning path based on OERs, and to teach what they learnt to other students using the learning path they created. The teacher role was to advise students more than to lead them. Emphasis was on peer collaboration and the students were provided some guidance to organize the group work through role playing. For collecting data on the emerging learning practices, the organizing team administrated a motivational survey at the beginning, and a final survey at the end of the laboratory. Questions were inspired by Ajzen's theory on the planned behavior (Ajzen, 1991), for the first survey. The final survey focused on attitude changes toward the workshop.

About qualitative data, the organizers worked as participant observers and wrote a report of the experience. Moreover, they organized a short interview some days after the workshop. In addition, the students were asked to fill in diaries during each collaborative session, in order to model the students' behaviour in the platform. Finally, in the surveys there were some open questions that gave organizers some further qualitative data to work with.

\subsection{Preliminary results}

Presently, we are analysing the qualitative and quantitative data, so we will here describe the preliminary findings. First, starting from the participant observation and from the diary data we have created a preliminary explorative model of the actions of the students within the platform, associated with the specific tools used. This model is guiding the refinement of the platform and it will be tested during the future editions of the workshop. For example, the action "search of OER" was carried out using the tools "OER search engine in 
CommonSpaces, google, google scholar, slideshare, youtube". This action was usually followed by the action "cataloguing OER" using the tool "OER cataloguing form". These two actions constituted an iterative cycle carried out many times, especially at the beginning of the workshop. The poster paper to be presented during the conference will contain a comprehensive scheme of this explorative model.

Moreover, we are analysing the surveys in order to grasp the students' ex-ante expectations and post-hoc perceptions concerning the elements of the workshop able to encourage their active participation. The results of this comparison show that throughout the laboratory there has been an increased interest in the topics of the laboratory. Although the number of students was too small, and do not allow any kind of generalization, some findings are interesting and relevant for future larger studies. While at the beginning $33 \%$ of the students mentioned the interest on the topic as a positive element for their participation, at the end $60 \%$ of the students expressed such interest. Moreover, $37 \%$ of the students expected that boredom would be an obstacle to their participation, but no students at the end of the course mentioned this aspect. Instead, the most cited negative element for the participation was that the workshop was too intensive for $65 \%$ of the students.

Finally, the interviews and the questionnaires reveal that some of the students struggled to engage in a teaching method they were not used to, giving hints for the design of the new edition of the laboratory. For example, most of the students used CommonSpaces for the cataloguing of OERs, ignoring its collaborative and social aspects, which are emphasized during the subsenquent editions of the workshop.

\section{Conclusion and future directions}

The challenge of designing a new "learning space", is to achieve a really Open Design. CommonSpaces has the ambition to be a three dimensional, open environment rather than a "platform". Into CommonSpaces and thanks to CommonSpaces, the learners and the community of learners should be able to reuse and create, plan and join others' learning proposal; and each one should be able and happy to trace and be traced in order to receive certification of her/his advancements in learning on some knowledge area. Many are the sub-challenges that we want to contribute to address with our effort of prototyping and experimenting :

1. to propose an aggregator, we may say a "meta-platform", using OER meta-data, capable to guide learners through many different authorities' modular Learning offer

2. to stimulate the emergence of new student-centered learning practices involving learners in programming and designing their own curriculum, exploiting the richness of the web; 
3. to stimulate higher education institutions to bet on the interoperability of their offers, giving to the community of learners a more active and meaningful role.

4. to propose a new model of tracking and assessment of learning activities partially based on the evaluation of individual contributions to a collaborative practice.

5. to propose a new model of collaborative learning in which each learner contribute to the Common Knowledge by means of "coding artefact", using "logic programming skills". With coding artefact we refer to regular expressions to build query or to cooperatively analyze corpora, logs, or transactions. In this sense, the concept of "programming skills" is proposed to learners, despite of their "computer literacy". Sharing of these codlet artefacts should not be considered a job for specialists but a kind of service for the community.

\section{References}

Ajzen, I., \& Cote, N. G. (2008). Attitudes and the prediction of behavior. In Crano, W.D. \& Prislin, R. (Ed.) Attitudes and attitude change, 289-311.

Berners-Lee, T., Hall, W., Hendler, J. A., O'Hara, K., Shadbolt, N., \& Weitzner, D. J. (2006). A framework for web science. Foundations and trends in Web Science, 1(1), 1130.

Hakkarainen, K. (2009). A knowledge-practice perspective on technology-mediated learning. International Journal of Computer-Supported Collaborative Learning, 4(2), 213-231.

Hakkarainen, K., \& Paavola, S. (2009). Toward a trialogical approach to learning. In B. Schwarz, T. Dreyfus, R. Hershkowitz (Eds.),Transformation of knowledge through classroom interaction, 65-80.

Ritella, G; Ligorio, M.B. \& Hakkarainen, K. (submitted) Theorizing space-time relations in education: the concept of chronotope.

Ritella, G., \& Hakkarainen, K. (2012). Instrumental genesis in technology-mediated learning: From double stimulation to expansive knowledge practices. International Journal of Computer-Supported Collaborative Learning, 7(2), 239-258.

Scardamalia, M. \& Bereiter, C. (2006). Knowledge building: Theory, pedagogy, and technology. In K. Sawyer (Ed.), Cambridge Handbook of the Learning Sciences. New York: Cambridge University Press.

Wenger, E., McDermott, R. A., \& Snyder, W. (2002). Cultivating communities of practice: A guide to managing knowledge. Harvard Business Press. 